\title{
Experimental Study on the Dielectric Model of Common Asphalt Pavement Surface Materials Based on the L-R Model
}

\author{
Yanhui Zhong $\mathbb{D}^{1},{ }^{1}$ Yanlong Gao, ${ }^{1}$ Bei Zhang $\mathbb{D}^{1},{ }^{1}$ Songtao Li $\mathbb{D},{ }^{2}$ Hongchuan Cui $\mathbb{D}^{3}{ }^{3}$ \\ Xiaolong $\mathrm{Li} \mathbb{D}^{1}{ }^{1}$ and Han $\mathrm{Zhao}^{3}$ \\ ${ }^{1}$ College of Water Conservancy and Environment, Zhengzhou University, 100 Science Rd., Zhengzhou 450001, China \\ ${ }^{2}$ Zhengzhou Railway Vocational \& Technical College, Zhengzhou 451460, China \\ ${ }^{3}$ Jilin Traffic Planning and Design Institute, Changchun 130021, China
}

Correspondence should be addressed to Bei Zhang; beizhang@126.com, Songtao Li; list16@126.com, and Hongchuan Cui; cui.hongchuan@163.com

Received 31 December 2020; Revised 25 March 2021; Accepted 13 April 2021; Published 27 May 2021

Academic Editor: Xun Xi

Copyright ( 2021 Yanhui Zhong et al. This is an open access article distributed under the Creative Commons Attribution License, which permits unrestricted use, distribution, and reproduction in any medium, provided the original work is properly cited.

\begin{abstract}
The establishment of a dielectric model of the asphalt pavement surface material is the premise and key to applying the electromagnetic wave technology to asphalt pavement nondestructive testing. Asphalt pavement can be made of different materials, including various types of asphalt mixtures. Therefore, in order to study and analyze the dielectric properties of different types of asphalt mixtures and establish a dielectric model of the asphalt pavement surface material, this paper studies four types of asphalt mixtures commonly used in the asphalt pavement surface course. Based on the comparative analysis of three classical models, the complex refractive index method (CRIM), Brown, and Looyenga; based on the L-R model, the linear regression analysis was conducted on the test data. The dielectric models which are suitable for the interpretation of four types of asphalt mixtures were established, and the dielectric model database of asphalt pavement surface materials was extended, which provides theoretical and technical support for nondestructive testing of the asphalt pavement.
\end{abstract}

\section{Introduction}

The composite dielectric model of asphalt mixtures describes the relationships between the dielectric constant of an asphalt mixture and its components, as well as the volume ratio [1]. The asphalt mixture is a commonly used pavement material. An asphalt mixture can be regarded as a mixed multiphase medium composed of air, asphalt, aggregate, and other three-phase media [2]. The establishment of the composite dielectric model of asphalt mixtures is the premise and key for nondestructive testing of the asphalt mixture by applying electromagnetic wave technology [3].

On the one hand, the dielectric properties of an asphalt mixture and the main factors that affect the dielectric properties of a mixed medium are analyzed in detail by using the dielectric models [4-11]. On the other hand, the volume ratio of each component of the asphalt mixture can be calculated using the measured dielectric constant of the asphalt mixture and further used for qualitative and quantitative analyses of density, porosity, and other parameters [12-17]. The establishment of a dielectric model of an asphalt mixture can provide the theoretical basis for nondestructive testing of the asphalt pavement $[18,19]$ and expand the application scope of electromagnetic testing technology significantly.

Some early scholars studied the dielectric behavior of composite materials and proposed different classic models, such as the Rayleigh model, Maxwell Garnett model, Böttcher model, and Clausius-Mossotti model [20-23]. These models are established on different assumptions, so they have their own limitation and certain application range. Thus, the main objective of this study is to improve the L- $\mathrm{R}$ model by using linear regression method and create a dielectric model suitable for explaining four specific types of asphalt mixture. 


\section{Materials and Methods}

In order to study the dielectric characteristics of the asphalt pavement surface materials, a dielectric model library of asphalt pavement surface materials was constructed. Two types of AC mixtures and two types of SMA mixtures were selected as research objects. As for AC mixtures, AC-13 and AC-16 were selected, and as for SMA mixtures, SMA-13 and SMA-16 were selected. The mineral aggregate grading of asphalt concrete mixtures is given in Table 1. The dielectric characteristics of the four types of asphalt mixtures were experimentally studied.

In this work, No. 70, No. 90, SBS-modified asphalt, and limestone were used to make the AC mixtures, and No. 70, SBS-modified asphalt, and basalt were used to make the SMA mixtures. The test materials are shown in Figure 1, and the basic physics of the single-phase media was in accordance with the specification requirements. The parameters were measured, and the measurement results showed that the material could meet the usage requirements.

A network analyzer was used to measure the dielectric constant of the single-phase medium using a coaxial probe method, as shown in Figure 2. The test frequency range of the single-phase dielectric material was $1.7 \mathrm{GHz}-2.4 \mathrm{GHz}$.

The four types of asphalt mixtures' oil-stone ratios were $3.9 \%, 4.0 \%, 4.1 \%, 4.9 \%, 5.0 \%, 5.1 \%, 5.9 \%, 6.0 \%$, and $6.1 \%$. First, a rut plate test piece was made according to the specifications. Then, a cutting machine was used to cut the rut plate test piece. There were 18 test pieces for each AC mixture, which was a total of $36 \mathrm{AC}$ test pieces, and 18 test pieces for each SMA mixture, so there were a total of 36 SMA mixture test pieces.

The network analyzer was used to measure the dielectric constant of the asphalt mixture test pieces using the waveguide method, as shown in Figure 3. The asphalt mixture test frequency range was also $1.6 \mathrm{GHz}-2.4 \mathrm{GHz}$.

After the dielectric-characteristic test of the asphalt mixture specimens was completed, the apparent density of each specimen was measured, and the volume ratio of each specimen component was calculated.

\section{Results}

3.1. Single-Phase Media. The coaxial probe method was used to measure the dielectric constant of the single-phase materials [8]. The measurement results are shown in Figure 4. Since the dielectric model in this paper does not consider the dielectric loss, only the real values of the dielectric constant are displayed in Figure 5. As shown in Figure 5, in the test frequency range, the dielectric constant of the single-phase medium was independent of the frequency. Therefore, the dielectric constant of the single-phase medium was the average value in the test frequency range, as shown in Table 2.

3.2. Asphalt Mixture. The waveguide method was adopted to measure the dielectric constant of the asphalt mixture specimens [24]. However, the frequency affected the dielectric properties of the asphalt mixtures. Due to the limited space, a set of test results is given Figure 4 . Within the test
TABLE 1: Mineral aggregate grading of asphalt concrete mixtures (square sieve).

\begin{tabular}{lcccc}
\hline \multirow{2}{*}{ Sieve hole $(\mathrm{mm})$} & \multicolumn{4}{c}{ Sieving rate (\%) } \\
& AC-13 & AC-16 & SMA-13 & SMA-16 \\
\hline $\mathbf{0 . 0 7 5}$ & 100 & 100 & 100 & 100 \\
$\mathbf{0 . 1 5}$ & 100 & 95.0 & 100 & 95.0 \\
$\mathbf{0 . 3}$ & 95.0 & 84.0 & 95.0 & 75.0 \\
$\mathbf{0 . 6}$ & 76.5 & 70.0 & 62.5 & 55.0 \\
$\mathbf{1 . 1 8}$ & 53.0 & 48.0 & 27.0 & 26.0 \\
$\mathbf{2 . 3 6}$ & 37.0 & 34.0 & 20.5 & 19.5 \\
$\mathbf{4 . 7 5}$ & 26.5 & 24.5 & 19.0 & 18.0 \\
$\mathbf{9 . 5}$ & 19.0 & 17.5 & 16.0 & 15.0 \\
$\mathbf{1 3 . 2}$ & 13.5 & 12.5 & 13.0 & 12.5 \\
$\mathbf{1 6}$ & 10.0 & 9.5 & 12.0 & 11.5 \\
$\mathbf{1 9}$ & 6.0 & 6.0 & 10.0 & 10.0 \\
\hline
\end{tabular}

frequency range, the dielectric constant of asphalt mixtures decreased with the increase in the test frequency.

Considering the limited space, this paper selects the 2.0 GHz test frequency as the representative and takes the dielectric constant of the asphalt mixture under this test frequency as the research object. The test results are shown in Figure 6. But the established model is also applicable to other test frequencies in the conclusions.

\section{L-R Model}

L-R model was proposed by Lichtenecker and Rother [25]. The relationship between the dielectric properties of a mixed multiphase medium and the dielectric properties of a singlephase media can be described by the L-R model, and it is given by

$$
\left(\varepsilon_{\mathrm{eff}}\right)^{\alpha}=\sum_{i=1}^{n} f_{i}\left(\varepsilon_{i}\right)^{\alpha},
$$

where the parameter denotes the fitting parameter of the relationship between the dielectric geometry and the dielectric constant, the value of $\alpha$ is in the range of $(-1,1), \varepsilon_{\text {eff }}$ denotes the dielectric constant of the medium, $\varepsilon_{i}$ is the dielectric constant of a phase $i$ of the medium, and $f_{i}$ is the volume ratio of a phase $i$ of the medium. The L-R model has been proven by theoretical analysis to be applicable to the interpretation of dielectric properties of a multiphase medium [21, 26-29] and has been expanded and applied in the field [30,31]. At $\alpha=1, \alpha=1 / 2$, and $\alpha=1 / 3$, the L-R model becomes the Brown [32], CRIM [33, 34], or Looyenga model [35], respectively. The expressions of the Brown, CRIM, and Looyenga models are, respectively, given by

$$
\begin{gathered}
\varepsilon_{\mathrm{eff}}=\sum_{i=1}^{n} f_{i} \varepsilon_{i}, \\
\sqrt{\varepsilon_{\mathrm{eff}}}=\sum_{i=1}^{n} f_{i} \sqrt{\varepsilon_{i}}, \\
\left(\varepsilon_{\mathrm{eff}}\right)^{1 / 3}=\sum_{i=1}^{n} f_{i}\left(\varepsilon_{i}\right)^{1 / 3} .
\end{gathered}
$$




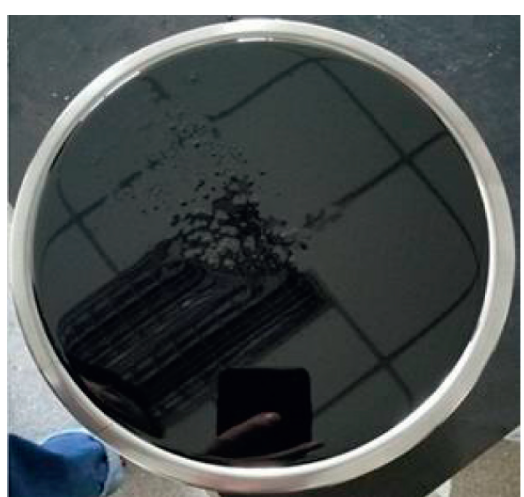

(a)

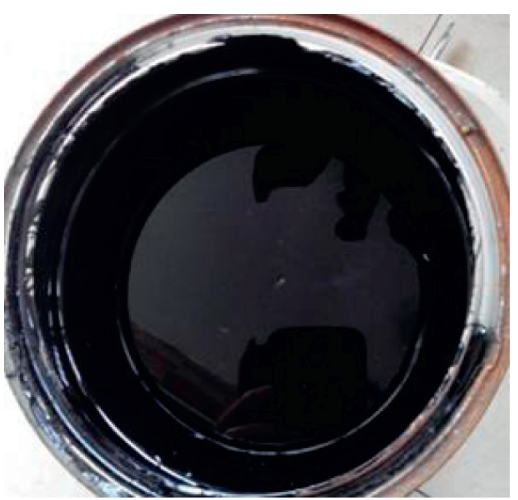

(b)

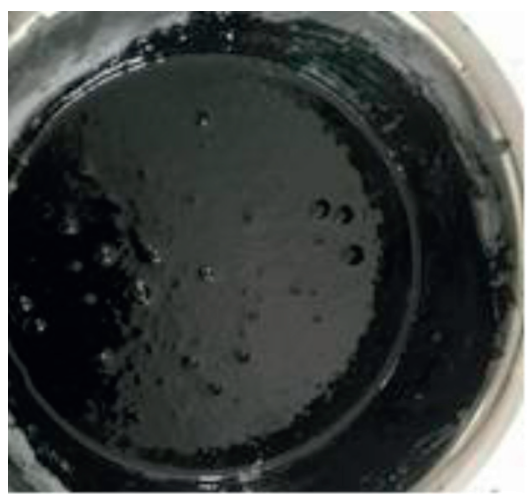

(c)

FIGURE 1: Test asphalts. (a) 70\# asphalt. (b) 90\# asphalt. (c) SBS-modified asphalt.

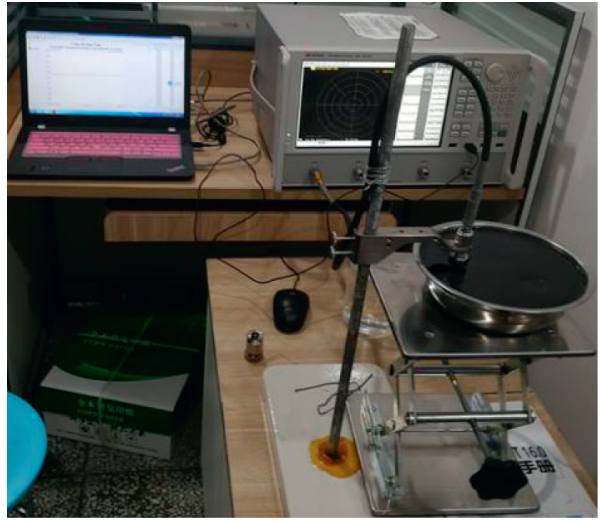

(a)

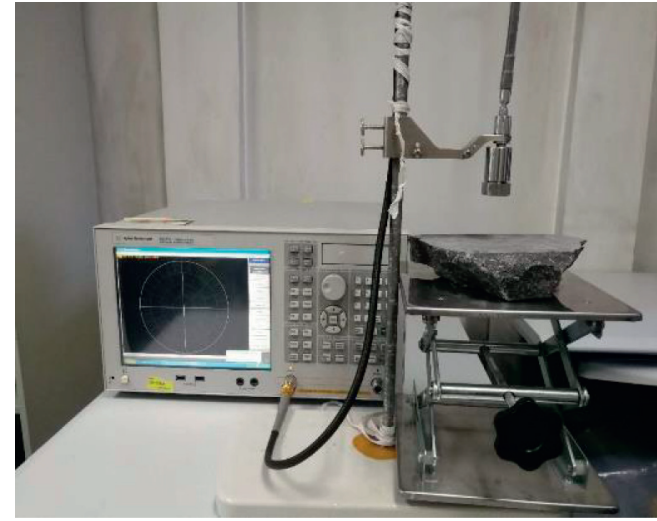

(b)

FIGURE 2: Single-phase material measurement process.

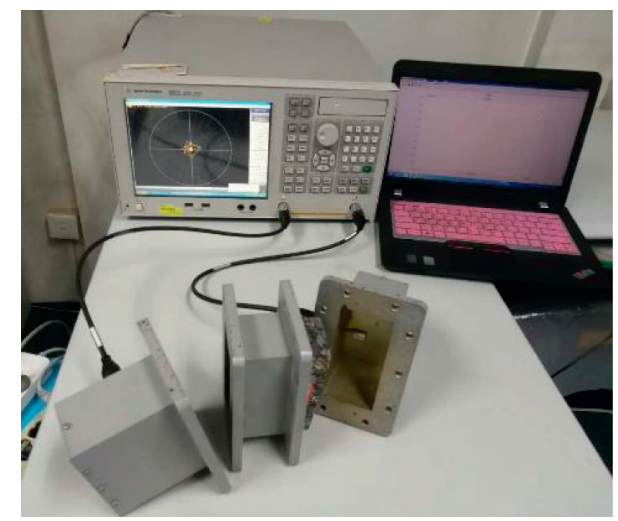

Figure 3: Dielectric constant measurement of the asphalt mixture test pieces.

In the above model, the meaning of each symbol is the same as in equation (1).

These models are widely used in soil physics, geophysics, oil logging, and other fields [30, 36-39]. However, these models experience some limitations in the application process [21, 22], especially in the description of dielectric properties of an asphalt mixture [14, 40-42].

The above three models are commonly used for the theoretical calculation of dielectric parameters of an asphalt mixture. Dielectric parameters of three single-phase materials are given in Table 2; the porosity of the asphalt mixture is set as $4 \%$, and the oil-stone ratio of the asphalt mixture is between $3 \%$ and $8 \%$.

The dielectric constants of the asphalt mixture were theoretically calculated using the Brown, CRIM, and Looyenga dielectric models, and the obtained results are shown in Figure 7.

Figure 7 shows that the results of the three models are different. The calculation result of the Brown model is the largest, while that of the Looyenga model is the smallest. All the three results decrease with the increase in the oil-stone ratio. The oil-stone ratio has an important effect on the dielectric properties of an asphalt mixture. The difference between the three results gradually increases with the increase in the oil-stone ratio. Therefore, it is needed to verify whether the three classic dielectric models can explain the dielectric properties of different types of asphalt mixtures. 


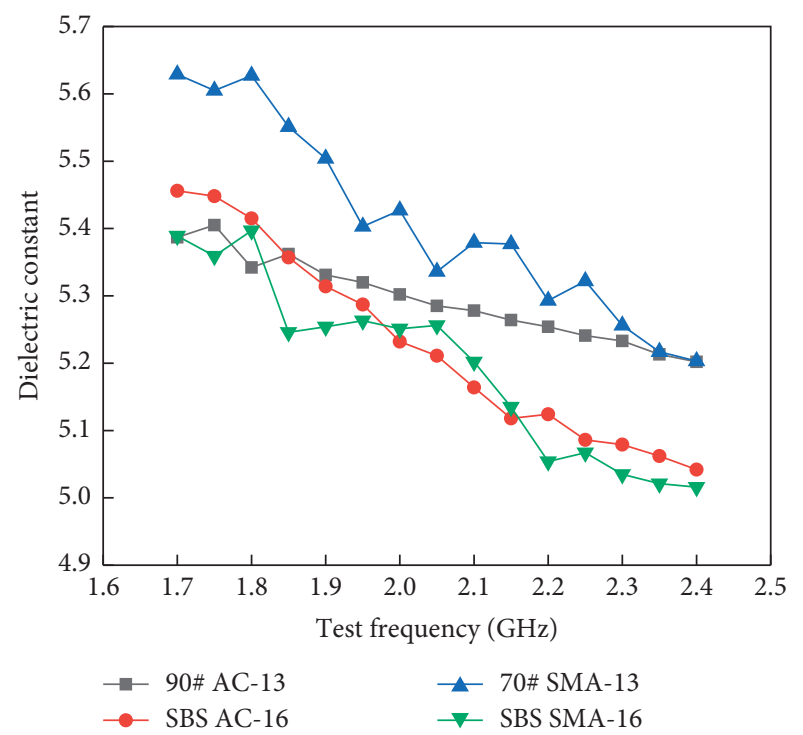

FIgURE 4: Dielectric constant of the asphalt mixtures.

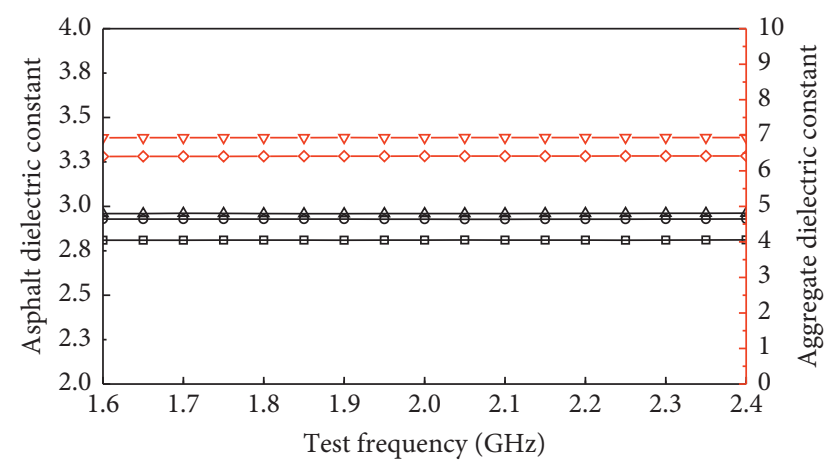

Asphalt dielectric constant Aggregate dielectric constant

$\begin{array}{ll}\rightarrow-70 \# & \rightarrow-\text { Basalt } \\ -90 \# & -\nabla-\text { Limestone } \\ \triangle \text { SBS } & \end{array}$

Figure 5: The dielectric constant of the single-phase materials.

TABLE 2: Average value of the dielectric constant of the single-phase materials.

\begin{tabular}{lcccc}
\hline $70 \#$ & $90 \#$ & SBS & Limestone block & Basalt stones \\
\hline 2.812 & 2.932 & 2.963 & 6.412 & 6.931 \\
\hline
\end{tabular}

\section{Discussion}

This paper studies four types of asphalt mixtures, including two types of asphalt concrete mixtures (ACs) and two types of asphalt mastic macadam mixtures (SMAs). By using the L-R model, on the basis of the research and analysis of the applicability of three classic dielectric models, the test data are linearly returned. Based on the regression analysis, a dielectric model is established to

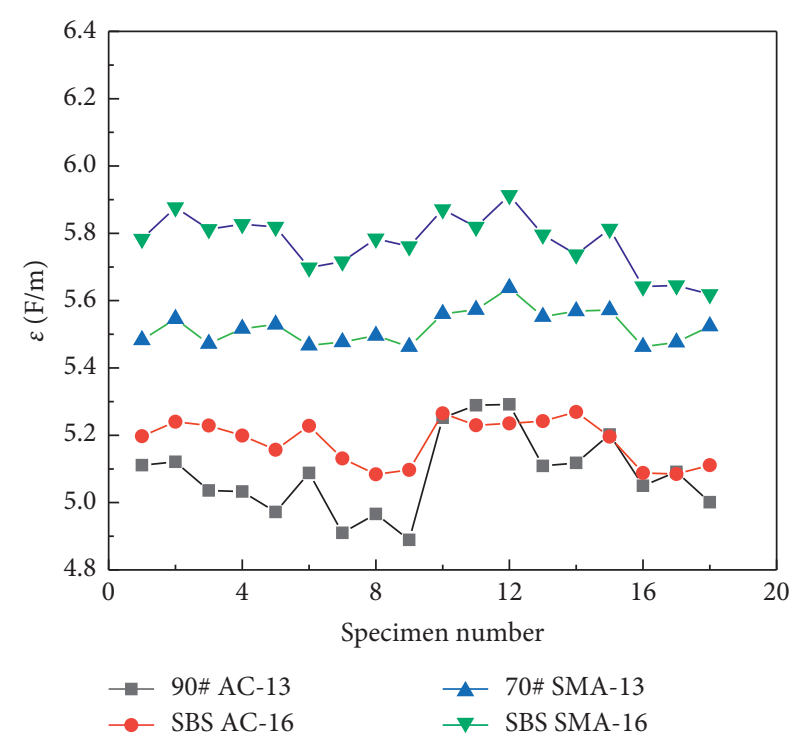

FIGURE 6: Dielectric constant of the asphalt mixtures at the test frequency of $2.0 \mathrm{GHz}$.

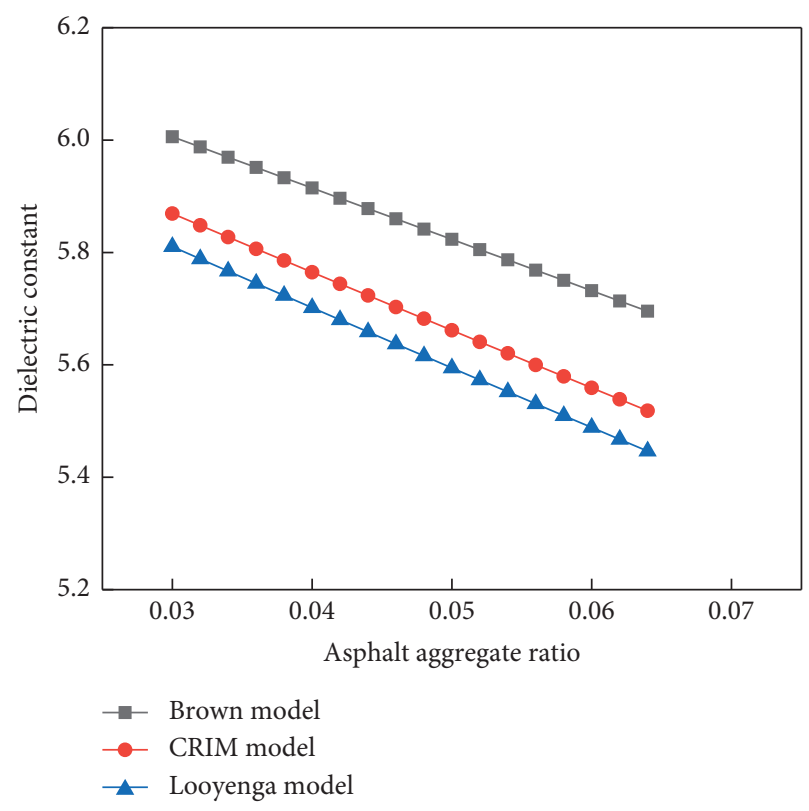

Figure 7: Theoretical calculation result.

explain the dielectric properties of four types of asphalt mixtures, forming the dielectric model database of asphalt pavement surface materials, which provides data support for the establishment of the dielectric model database of the pavement material and important theoretical support for the inversion of the asphalt mixture multiphase volume fraction.

5.1. Applicability Analysis of the Classical Dielectric Model. Three classic dielectric models, namely, Brown, CRIM, and Looyenga, were used to analyze the test results, and the 
calculated values were compared with the test values. The error was calculated by

$$
\begin{aligned}
& \eta=\frac{E-A}{E / 100}, \\
& \theta=\sum_{i=1}^{n} \frac{\eta_{i}}{n}
\end{aligned}
$$

where $\eta$ denoted the calculation error, $A$ denoted the theoretical value, $E$ denoted the actual measured value, and $\theta$ denoted the average error.

The obtained analysis results are shown in Figures 8-10.

The aggregate had a higher dielectric constant and the largest volume ratio. Therefore, the aggregate had the largest influence on the dielectric constant of the asphalt mixture, and the aggregate particle size was large. For the CRIM model, the model particle size was smaller than that the wavelength could meet. The Brown and Looyenga models have been often used to explain the dielectric properties of two-phase media, such as soil. Therefore, under the current value of $c$, the dielectric constant error of the asphalt mixture calculated using the three classic dielectric models was large, so these three dielectric models were not applicable. Thus, in order to explain the dielectric characteristics of the abovementioned asphalt mixtures, it is necessary to establish dielectric models for different types of asphalt mixtures, namely, different dielectric models should be used to explain the dielectric characteristics of different types of asphalt mixtures.

\subsection{Establishment and Verification of the Dielectric Model of} the Common Asphalt Mixture. The applicability of a dielectric model is the key to the nondestructive testing of an asphalt mixture using the ground-penetrating radar. The above results show that three classic dielectric models are not suitable for explaining the dielectric characteristics of an asphalt mixture. Therefore, based on the L-R formula, using the binary linear regression analysis method, the dielectric properties' test results of different types of asphalt mixture are analyzed, and the $C$ value of different types of asphalt mixture corresponding to the $\mathrm{L}-\mathrm{R}$ equation is obtained. The binary linear regression analysis is carried out on the $\mathrm{L}-\mathrm{R}$ model under each $C$ value, and the $F$ test is carried out on the regression analysis results to check whether the binary linear regression results are correct. The square sum of error $\left(e^{2}=\sum\left(\varepsilon_{m}-\overline{\varepsilon_{x}}\right)^{2}\right)$ between the theoretical value and the experimental value of the dielectric constant of the asphalt mixture under the current model is calculated. The analysis results of AC-13, AC-16, SMA-13, and SMA-16 are shown in Table 3.

Based on the above analysis results, the $c$ value corresponding to the minimum square sum of the error between the theoretical value calculated by the dielectric model and the experimental value of the dielectric properties of the asphalt mixture is the best dielectric model of this kind of asphalt mixture, and they are given by

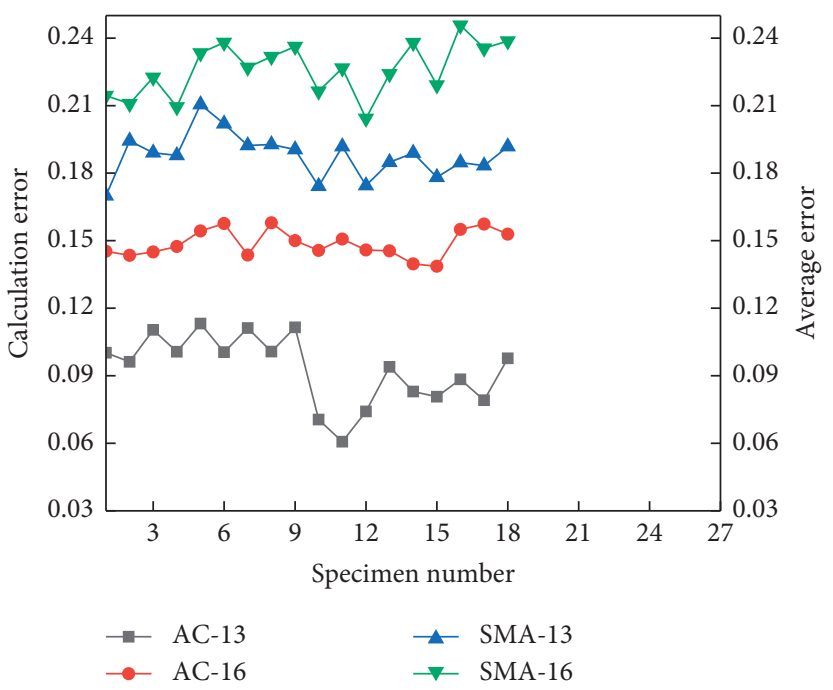

Figure 8: Applicability analysis of the Brown model.

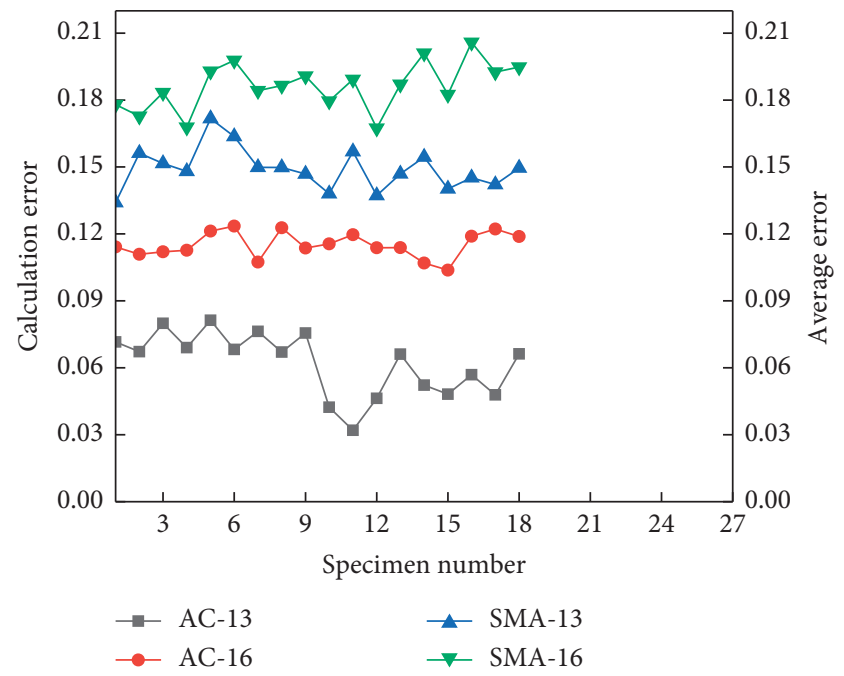

FIgURE 9: Applicability analysis of the CRIM model.

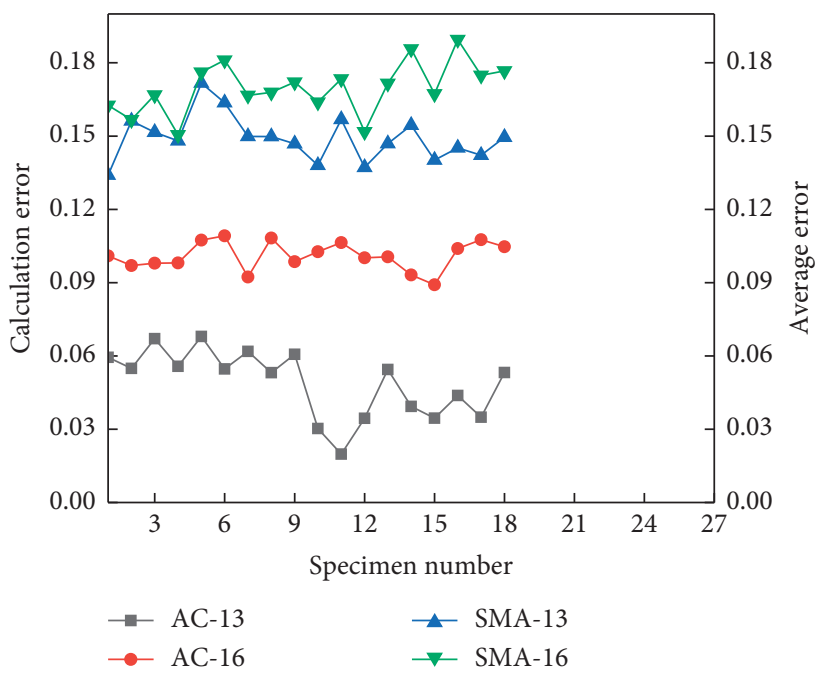

Figure 10: Applicability analysis of the Looyenga model. 
TABLE 3: Linear regression statistical data of four asphalt concrete types.

\begin{tabular}{|c|c|c|c|c|c|c|c|c|c|c|c|}
\hline \multicolumn{3}{|c|}{ AC-13 } & \multicolumn{3}{|c|}{ AC-16 } & \multicolumn{3}{|c|}{ SMA-13 } & \multicolumn{3}{|c|}{ SMA-16 } \\
\hline$c$ & $F$ & $e^{2}$ & $c$ & $F$ & $e^{2}$ & $c$ & $F$ & $e^{2}$ & C & $F$ & $e^{2}$ \\
\hline-1 & 25.60 & 2.95 & -1 & 26.46 & 1.57 & -1 & 15.09 & 14.01 & -1 & 15.22 & 9.91 \\
\hline-0.9 & 27.22 & 1.44 & 0.9 & 31.16 & 0.53 & -0.9 & 15.12 & 10.86 & -0.9 & 15.29 & 7.30 \\
\hline-0.8 & 33.07 & 0.50 & 0.8 & 95.87 & 0.05 & -0.8 & 15.16 & 8.22 & -0.8 & 15.41 & 5.18 \\
\hline-0.7 & 98.91 & 0.06 & -0.7 & 76.96 & 0.07 & -0.7 & 15.22 & 6.05 & -0.7 & 15.59 & 3.50 \\
\hline-0.6 & 110.43 & 0.05 & -0.6 & 30.88 & 0.51 & -0.6 & 15.30 & 4.30 & -0.6 & 15.91 & 2.21 \\
\hline-0.5 & 34.17 & 0.41 & -0.5 & 26.59 & 1.30 & -0.5 & 15.44 & 2.92 & -0.5 & 16.54 & 1.26 \\
\hline-0.4 & 27.73 & 1.06 & -0.4 & 25.36 & 2.39 & -0.4 & 15.66 & 1.87 & -0.4 & 18.03 & 0.62 \\
\hline-0.3 & 25.93 & 1.96 & -0.3 & 24.84 & 3.70 & -0.3 & 16.08 & 1.10 & -0.3 & 22.90 & 0.23 \\
\hline-0.2 & 25.19 & 3.05 & -0.2 & 24.58 & 5.18 & -0.2 & 17.01 & 0.57 & -0.2 & 48.07 & 0.05 \\
\hline-0.1 & 24.81 & 4.28 & -0.1 & 24.42 & 6.80 & -0.1 & 19.51 & 0.24 & -0.1 & 44.47 & 0.06 \\
\hline 0.1 & 24.45 & 7.03 & 0.1 & 24.25 & 10.25 & 0.1 & 28.83 & 0.07 & 0.1 & 18.07 & 0.48 \\
\hline 0.2 & 24.35 & 8.48 & 0.2 & 24.21 & 12.02 & 0.2 & 10.18 & 0.17 & 0.2 & 16.63 & 0.84 \\
\hline 0.3 & 24.28 & 9.94 & 0.3 & 24.17 & 13.80 & 0.3 & 17.23 & 0.36 & 0.3 & 16.01 & 1.28 \\
\hline 0.4 & 24.23 & 11.42 & 0.4 & 24.14 & 15.57 & 0.4 & 16.17 & 0.63 & 0.4 & 15.68 & 1.78 \\
\hline 0.5 & 24.20 & 12.88 & 0.5 & 24.12 & 17.31 & 0.5 & 15.70 & 0.96 & 0.5 & 15.49 & 2.32 \\
\hline 0.6 & 24.17 & 14.31 & 0.6 & 24.11 & 19.00 & 0.6 & 15.46 & 1.33 & 0.6 & 15.36 & 2.90 \\
\hline 0.7 & 24.14 & 15.72 & 0.7 & 24.09 & 20.66 & 0.7 & 15.32 & 1.74 & 0.7 & 15.28 & 3.49 \\
\hline 0.8 & 24.13 & 17.09 & 0.8 & 24.08 & 22.26 & 0.8 & 15.22 & 2.17 & 0.8 & 15.22 & 4.09 \\
\hline 0.9 & 24.11 & 18.42 & 0.9 & 24.07 & 23.80 & 0.9 & 15.16 & 2.62 & 0.9 & 15.18 & 4.71 \\
\hline 1 & 24.10 & 19.70 & 1 & 24.07 & 25.29 & 1 & 15.12 & 3.08 & 1 & 15.15 & 5.32 \\
\hline
\end{tabular}

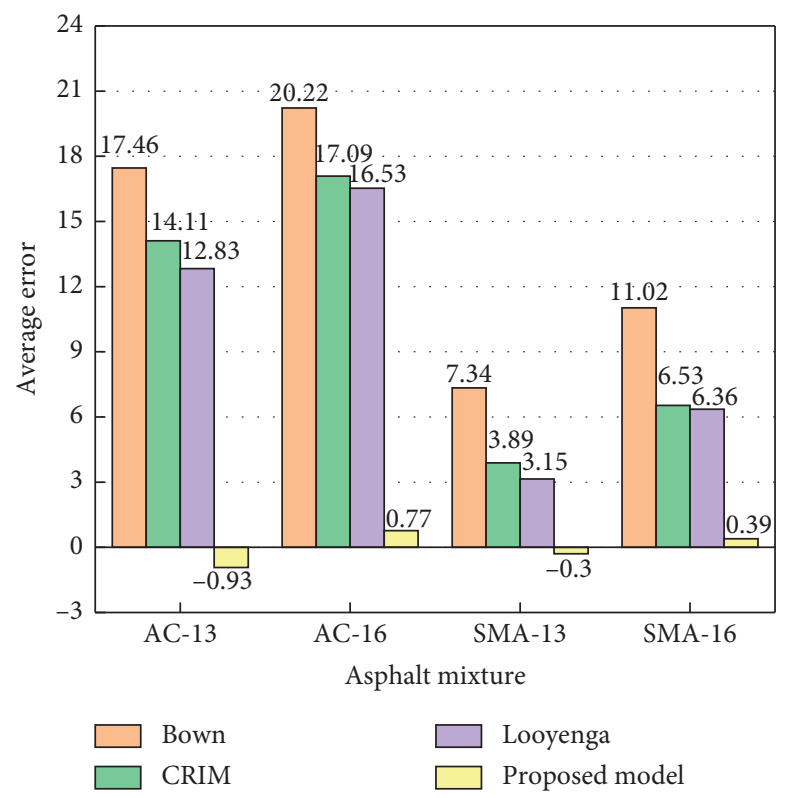

FIGURE 11: Average error of the classical model and the proposed models.

$$
\begin{aligned}
& \left(\varepsilon_{\mathrm{ac}-13}\right)^{-0.6}=f_{g}\left(\varepsilon_{g}\right)^{-0.6}+f_{l}\left(\varepsilon_{l}\right)^{-0.6}+f_{a}\left(\varepsilon_{a}\right)^{-0.6}, \\
& \left(\varepsilon_{\mathrm{ac}-16}\right)^{-0.8}=f_{g}\left(\varepsilon_{g}\right)^{-0.8}+f_{l}\left(\varepsilon_{l}\right)^{-0.8}+f_{a}\left(\varepsilon_{a}\right)^{-0.8}, \\
& \left(\varepsilon_{\mathrm{sma}-13}\right)^{0.1}=f_{g}\left(\varepsilon_{g}\right)^{0.1}+f_{l}\left(\varepsilon_{l}\right)^{0.1}+f_{a}\left(\varepsilon_{a}\right)^{0.1}, \\
& \left(\varepsilon_{\mathrm{sma}-16}\right)^{-0.2}=f_{g}\left(\varepsilon_{g}\right)^{-0.2}+f_{l}\left(\varepsilon_{l}\right)^{-0.2}+f_{a}\left(\varepsilon_{a}\right)^{-0.2} .
\end{aligned}
$$

Figure 11 shows the average error of the four classical models and the established models. As can be seen from the figure, the new model has the highest accuracy. The established models were used to verify the test results. The calculation results are shown in Figure 12. The results show that the overall calculation accuracy is within $3 \%$, which meets the usage requirements.

In this work, the dielectric properties of the common asphalt mixture for the asphalt pavement surface were studied. The Brown, CRIM, and Looyenga dielectric models were used to explain the dielectric properties of the four types of asphalt mixtures. The comprehensive test and calculation results show that Brown, CRIM, and Looyenga dielectric models are not suitable to explain the dielectric properties of the four types of asphalt mixtures. 


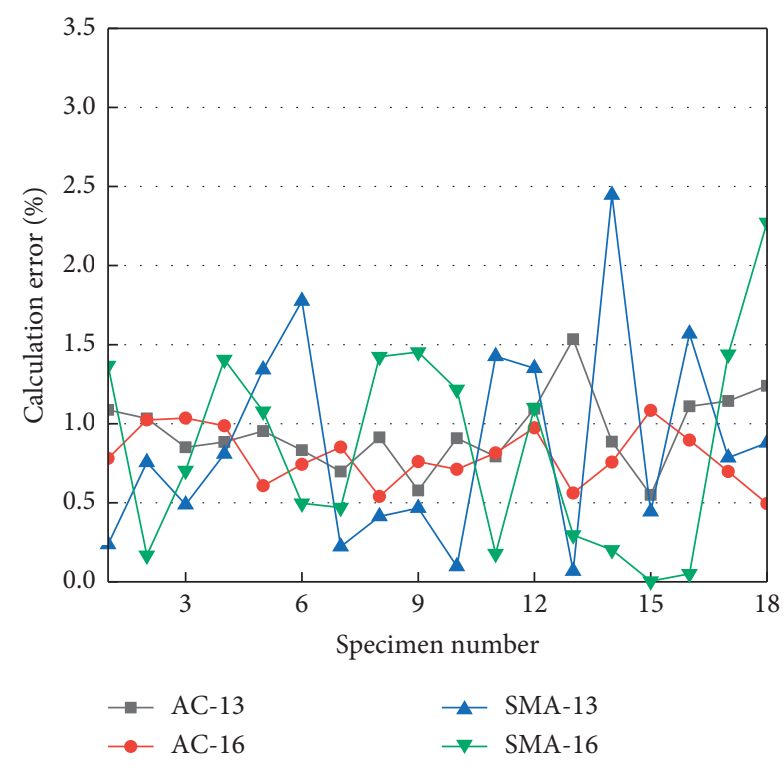

Figure 12: The results of the established models.

\section{Conclusions}

In this study, based on the linear regression analysis of the L-R equation and test data, the author establishes four dielectric models of asphalt mixtures. Based on the results of this study, the following conclusions can be drawn:

(1) Compared with the calculation results of the classic L- $\mathrm{R}$ model, it is obvious that the four models established have higher calculation accuracy and are more in line with the actual measurement results

(2) According to the established dielectric model, the error in the result of calculating the volume ratio of each component by measuring the dielectric constant of the asphalt mixture can be made smaller

(3) The established model has been verified to be suitable for explaining the dielectric properties of asphalt mixtures, enriching the dielectric model database of asphalt pavement surface materials

(4) The constructed four dielectric models of asphalt mixtures can guide the nondestructive testing of the asphalt pavement surface and provide support for the nondestructive testing and maintenance of the asphalt pavement

(5) Based on the examination of the rationality of the existing classic dielectric models to explain the dielectric properties of asphalt mixtures, this paper establishes four dielectric models for commonly used asphalt mixtures, which provides data for the establishment of a pavement material dielectric model library stand by.

\section{Data Availability}

All data, models, and codes generated or used during the study are included within this article.

\section{Conflicts of Interest}

The authors declare that they have no conflicts of interest.

\section{Acknowledgments}

The research presented in this work was supported in part by the National Key Research and Development Projects (Grant no. 2018YFB1600200), National Natural Science Foundation of China (Grant nos. 51878624 and 51878622), Henan Science Fund for Distinguished Young Scholars (Grant no. 202300410354), Major Scientific and Technological Special Project in Henan (Grant no. 181100310400), Program for Science and Technology Innovation Talents in Universities of Henan Province (Grant no. 19HASTIT041), and Key Research Projects of Higher Education in Henan Province (Grant no. 18A580001).

\section{References}

[1] B. Zhang, System Identification Method for Backcalculating the Dielectric Property and Thickness of Pavement StructuresStudy on Applied Technology of Ground Penetrating Radar, Chongqing University, Chongqing, China, 2013.

[2] P. Subedi and I. Chatterjee, "Dielectric mixture model for asphalt-aggregate mixitures," Journal of Microwave Power and Electromagnetic Energy, vol. 28, no. 2, pp. 68-72, 1993.

[3] M. Meng, Study on Dielectric Model Including the Frequency and Temperature of Concrete and Asphalt Mixture, Zhengzhou University, Zhengzhou, China, 2014.

[4] I. Amine, F. Bosc, B. Guan et al., "Full-waveform inversion using a stepped-frequency GPR to characterize the tack coat in hot-mix asphalt (HMA) layers of flexible pavements," NDT \& E International, vol. 95, pp. 17-25, 2018.

[5] R. Evans, M. Frost, M. Stonecliffe-Jones, and N. Dixon, "Assessment of in situ dielectric constant of pavement materials," Transportation Research Record: Journal of the Transportation Research Board, vol. 2037, no. 1, pp. 128-135, 2007.

[6] J. Edward, J. G. Jaselskis, and A. Brilingas, "Dielectric properties of asphalt pavement," Journal of Materials in Civil Engineering, vol. 15, no. 5, pp. 427-434, 2003.

[7] W. L. Lai, W. F. Tsang, H. Fang et al., "Experimental determination of bulk dielectric properties and porosity of porous asphalt and soils using GPR and a cyclic moisture variation technique," Geophysics, vol. 71, no. 4, pp. K93-K102, 2006.

[8] E. J. Jaselskis, J. Grigas, and A. Brilingas, "Dielectric properties of asphalt pavement," Journal of Materials in Civil Engineering, vol. 15, no. 5, pp. 427-434, 2003.

[9] J. Q. Shang, "Effects of asphalt pavement properties on complex permittivity," International Journal of Pavement Engineering, vol. 3, no. 4, pp. 217-226, 2002.

[10] T. Saarenketo, Electrical properties of road materials and subgrade soils and the use of ground penetrating radar in traffic infrastructure surveys, Ph.D. Thesis, University of Oulu, Oulu, Finland, 2006.

[11] J. Qin, J. Li, V. Lee, H. Jaeger, J. J. de Pablo, and K. F. Freed, “A theory of interactions between polarizable dielectric spheres," Journal of Colloid And Interface Science, vol. 469, pp. 237-241, 2016.

[12] F. M. Fernandes, A. Fernandes, and J. Pais, "Assessment of the density and moisture content of asphalt mixtures of road 
pavements," Construction \& Building Materials, vol. 154, pp. 1-10, 2017.

[13] Z. Leng, I. L. Al-Qadi, and S. Lahouar, "Development and validation for in situ asphalt mixture density prediction models," NDT \& E International, vol. 44, no. 4, pp. 369-375, 2011.

[14] C.-M. Chang, J.-S. Chen, and T.-B. Wu, "Dielectric modeling of asphalt mixtures and relationship with density," Journal of Transportation Engineering, vol. 137, no. 2, pp. 104-111, 2011.

[15] A. Turesson, "Water content and porosity estimated from ground-penetrating radar and resistivity," Journal of Applied Geophysics, vol. 58, no. 2, pp. 99-111, 2006.

[16] X. Yu, Research on Dielectric Constant of Asphalt Mixture, Wuhan University of Technology, Wuhan, China, 2018.

[17] Z. Wang, Y. Cai, and C. Guo, "Ground penetrating radar experimental study on density and compaction of asphalt pavement," Central South Highway Engineering, no. 4, pp. 96-98+101, 2006.

[18] Y. Zhai, Research on Dielectric Properties of Asphalt Mixtures Based on Multi-Scale Analysis and Micromechanics Modeling, Zhengzhou University, Zhengzhou, China, 2019.

[19] F. M. Wang, B. Zhang, Y. C. Cai, and Y. H. Zhong, Inversion Theory and Application of Layered System Dielectric Property, Science Press, Beijing, China, 2011.

[20] R. L. Lvi, "On the influence of obstacles arranged in rectangular order upon the properties of a medium," Philosophical Magazine, vol. 34, no. 211, pp. 481-502, 1976.

[21] J. C. M. Garnett, "Colours in metal glasses and in metallic films," Philosophical Transactions of the Royal Society of London, vol. 203, pp. 385-420, 1904.

[22] C. J. F. Böttcher, "The dielectric constant of crystalline powders," Recueil des Travaux Chimiques des Pays-Bas.vol. 64, no. 2, pp. 47-51, 2015.

[23] H. E. J. Neugebauer, "Clausius-mosotti equation for certain types of anisotropic crystals," Canadian Journal of Physics, vol. 32, no. 1, pp. 1-8, 1954.

[24] B. Zhang, Y. H. Zhong, H. X. Liu, and F. M. Wang, "Experimental research on dielectric constant model for asphalt concrete material," Advanced Materials Research, vol. 250-253, pp. 2760-2764, 2011.

[25] J. G. Liu, "Measuring electrostatic force and permittivity of vacuum by an electronic balance," Physics Experimentation, 2003.

[26] J. Xu, Q. Chen, and E. Ni, "Research on the method and the theory of measuring permittivity of microwave ceramics by cutoff waveguide dielectric resonator," Chinese Journal of Scientific Instrument, vol. 27, no. 10, pp. 1322-1325, 2006.

[27] K. Lichtenecker and K. Rother, "Die herleitung des logarithmischen mischungs-gesetzes aus allegemeinen prinzipien der staionaren stromung," Physikalische Zeitschrift, vol. 32, pp. 255-260, 1931.

[28] K. Roth, R. Schulin, H. Flühler, and W. Attinger, "Calibration of time domain reflectometry for water content measurement using a composite dielectric approach," Water Resources Research, vol. 26, no. 10, pp. 2267-2273, 1990.

[29] M. Dobson, F. Ulaby, M. Hallikainen, and M. El-rayes, "Microwave dielectric behavior of wet soil-part ii: dielectric mixing models," IEEE Transactions on Geoscience and Remote Sensing, vol. GE-23, no. 1, pp. 35-46, 1985.

[30] A. M. Shutko and E. M. Reutov, "Mixture formulas applied in estimation of dielectric and radiative characteristics of soils and grounds at microwave frequencies," IEEE Transactions on Geoscience and Remote Sensing, vol. GE-20, no. 1, pp. 29-32, 1982.
[31] T. Zakri, J.-P. Laurent, and M. Vauclin, "Theoretical evidence for "Lichtenecker's mixture formulae" based on the effective medium theory," Journal of Physics D: Applied Physics, vol. 31, no. 13, pp. 1589-1594, 1998.

[32] H. Looyenga, "Dielectric constants of heterogeneous mixtures," Physica, vol. 31, no. 3, pp. 401-406, 1965.

[33] C. J. F. Böttcher, "The dielectric constant of crystalline powders," Recueil des Travaux Chimiques des Pays-Bas, vol. 64, no. 2, pp. 47-51, 2015.

[34] W. F. Brown, Dielectrics in Encyclopedia of Physics, pp. 15-16, Springer, Berlin, Germany, 1956.

[35] J. R. Brichak, C. G. Gardner, J. E. Hipp, and J. M. Victor, "High dielectric contant microwave probes for sensing soil moisture," Proceedings of the IEEE, vol. 62, no. 1, pp. 93-98, 1974.

[36] R. P. Wharton, G. A. Hazen, R. W. Rau et al., "Advances in technique and interpretation," in Proceedings of the 55th Annual Technical Conference and Exhibition, Dallas, TX, USA, September 1980.

[37] L. D. Landau and E. M. Lifshitz, Electrodynamics of Continuous Media, Pergamon Press, Oxford, UK, 1960.

[38] J. Zhang and D. Zhang, "Microwave remote sensing field measurement of dielectric constant of typical objects," Journal of Electronics \& Information Technology, vol. 19, no. 4, pp. 566-569, 1997.

[39] Q. Feng, X. I. Li, and H. Zheng, "Experimental study of $1 \mathrm{kHz}-15 \mathrm{MHz}$ rock dielectric constant," Chinese Journal of Geophysics, vol. 38, no. 1, pp. 331-336, 1995.

[40] S. Song, "Study on microwave dielectric properties of coastal soil," Chinese Journal of Geophysics, vol. 38, no. 1, pp. 331-336, 1995.

[41] W. Wang and Y. Yang, "Experimental study of rock permittivity of daqing oilfield," Well Logging Technology, no. 6, p. 219, 1993.

[42] A. V. Goncharenko, V. Z. Lozovski, and E. F. Venger, "Lichtenecker's equation: applicability and limitations," Optics Communications, vol. 174, no. 1-4, pp. 19-32, 2000. 